\title{
IDENTIFICATION AND COMPENSATION OF THE ELECTRODYNAMIC TRANSDUCER NONLINEARITIES
}

\author{
Hans Schurer ${ }^{1}$, Alex G.J. Nijmeijer ${ }^{2}$, Mark A. Boer ${ }^{2}$, Cornelis H. Slump ${ }^{1}$ and Otto E. Herrmann ${ }^{1}$ \\ ${ }^{1}$ University of Twente, Department of Electrical Engineering, \\ Laboratory for Network Theory and VLSI-Design, \\ P.O. box 217, 7500 AE Enschede, The Netherlands \\ ${ }^{2}$ AEMICS B.V., H.C. Pootstraat 24, 7552 WN Hengelo, The Netherlands \\ E-mail: hans@nt.el.utwente.nl.
}

\begin{abstract}
Based on a simplified nonlinear lumped element model of the electrodynamic loudspeaker in either a closed or a vented cabinet, a new nonlinear controller is derived, simulated and implemented on a DSP. The Volterra series expansion, a well known functional expansion to model nonlinear systems, is used to estimate the nonlinear parameters from distortion measurements. The controller is directly based on the nonlinear differential equation, and is tested for the case of a low frequency electrodynamic loudspeaker in a closed cabinet. Digital implementation is realized on a general purpose TMS320C30 DSP development board, using the automatic code generation from schematic entry of the Alta-Group SPW software.
\end{abstract}

\section{INTRODUCTION}

The electrodynamic loudspeaker suffers from several nonlinearities of which the most important ones are displacement $(x)$ dependent. Improvement of electro-acoustic transduction behavior can of course be achieved by changing the electrical, magnetic, mechanical and acoustical design of the transducer. These optimizations lead mostly to a more expensive product and the question arises whether the nonlinearities can be reduced in another manner. With the ever decreasing prices of digital signal processing hardware, linearization of the transducer by means of an algorithm implemented on a DSP becomes feasible. Besides this, linear equalizing of loudspeaker systems is already increasingly applied using digital signal processing techniques.

\section{SYSTEM IDENTIFICATION}

\subsection{Lumped element model}

The major physical causes for the nonlinear transduction in electrodynamic loudspeakers appear to be:

- The displacement dependent mechanical stiffness of the suspension $k_{t}(x)$

- The displacement dependent electro-mechanic transduction factor, called the force factor $\mathrm{Bl}(\mathrm{x})$

- The displacement dependent self-inductance of the voice coil $L_{e}(x)$

The first one is caused by material properties of the suspension. The latter two by the movement of the voice coil out of the radial magnetic field and the movement of the magnetic core inside the voice coil, respectively.

Based on the well known lumped element approach for system modeling we obtain the two coupled nonlinear differential equations for a voltage driven loudspeaker, in either a closed or a vented cabinet

$$
\begin{aligned}
u_{e} & =R_{e} i+\frac{d L_{e}(x) i}{d t}+B l(x) \frac{d x}{d t} \\
B l(x) i & =\mathcal{L}^{-1}\{Z(s)\} * x+k_{t}(x) x-\frac{i^{2}}{2} \frac{d L_{e}(x)}{d x}
\end{aligned}
$$

where $\mathcal{L}^{-1}\{\}$ denotes the inverse Laplace transform, * convolution, $u_{e}$ is the driving voltage at the terminals and $i$ the voice coil current. The last term in Eq.(2) is the reluctance force which is caused by the displacement dependent magnetic energy, which on its turn is caused by the selfinductance nonlinearity. The linear element in the electrical domain is the voice coil resistance $R_{e}$. Linear elements in the mechanical domain are taken together in the mechanical impedance $Z(s)$, which is given by

$$
\begin{aligned}
& Z(s)=m_{t} s^{2}+R_{m} s \\
& Z(s)=m_{t} s^{2}+R_{m} s+\frac{s^{2} m_{a p} S_{d}^{2}}{s^{2} m_{a p} c_{a}+1}
\end{aligned}
$$

for respectively a speaker in a closed cabinet Eq.(3), and in a vented cabinet Eq. (4). Elements in this impedance are the effective mass $m_{t}$, mechanical damping $R_{m}$, mass of the moving air in the vent $m_{a p}$, compliance of the air in the cabinet $c_{a}$ and the diaphragm surface area $S_{d}$. Note that the form of the impedance $Z(s)$ is the only difference between the differential equations for the vented and closed cabinet case. This will simplify the derivation of a linearizing controller as we will see later.

Nonlinear elements are described by truncated Taylor series expansions in $x$. The parameters of these expansions are denoted as the nonlinear parameters of the model. The linear parameters are determined from input impedance and sound pressure response measurements. The nonlinear parameters are determined by optimization of the Volterra frequency domain kernels, which are introduced next.

\subsection{Volterra series}

From the nonlinear differential equation, found by substitution of Eq.(2) in Eq.(1), we derive a nonlinear model in the s-domain, based on the Volterra series expansion. In general the output $y(t)$ of a nonlinear system, characterized by the continuous time Volterra series, is given by

$$
\begin{array}{r}
y(t)=h_{0}+\int_{-\infty}^{\infty} h_{1}(\tau) x(t-\tau) d \tau+ \\
\int_{-\infty}^{\infty} \int_{-\infty}^{\infty} h_{2}\left(\tau_{1}, \tau_{2}\right) x\left(t-\tau_{1}\right) x\left(t-\tau_{2}\right) d \tau_{1} \tau_{2}+\ldots
\end{array}
$$


where $x(t)$ is the system input and $h_{n}\left(\tau_{1}, \ldots, \tau_{n}\right)$ are the generalized impulse responses, also called kernels. Similar to linear systems we can determine, using the multidimensional Laplace transform, the response in the sdomain (using $s=\sigma+j \omega$ the complex frequency variable)

$$
\begin{aligned}
Y(s)= & H_{1}(s) X(s)+\Gamma\left\{H_{2}\left(s_{1}, s_{2}\right) X\left(s_{1}\right) X\left(s_{2}\right)\right\}+ \\
& \Gamma^{2}\left\{H_{3}\left(s_{1}, s_{2}, s_{3}\right) X\left(s_{1}\right) X\left(s_{2}\right) X\left(s_{3}\right)\right\} \ldots
\end{aligned}
$$

where the capital letters denote the Laplace transformed versions of their small letter counterparts and $\Gamma^{n}\{\}$ the contraction operator. The system response is thus determined by a summation of all kernel responses, i.e. the Volterra series can be seen as a Taylor series with memory [1].

The s-domain versions of the linear kernel $H_{1}$ and nonlinear kernels $\mathrm{H}_{2}$ and $\mathrm{H}_{3}$ are derived (using the harmonic balance method) from the nonlinear differential equation driven with a multi-tone excitation [2].

Nonlinear parameters are determined by optimization of these Volterra s-domain kernels on distortion measurements, using a Simplex search method [3], minimizing

$$
\max _{s_{i}}|| Y_{\text {measured }}\left(s_{i}\right)|-| Y_{\text {model }}\left(s_{i}\right)||
$$

with $Y_{\text {model }}(s)$ the complex output of the model given by Eq. (6), and $Y_{\text {measured }}(s)$ the magnitude of the measured distortions. Final results of this optimization are given in Fig. 1. Note that the distortions by the model are under-estimated, especially for frequencies in the range of $100-200 \mathrm{~Hz}$. This is less a problem than in case of overestimation, which leads to an increase of distortions. Next to this, distortions in this frequency span are already relatively low. Synthesis of a linearizing controller based on the Volterra description is also possible, but for real-time implementation limited to compensation of second order distortions and not considered here [4].

\section{NONLINEAR CONTROLLER}

The controller which is considered here is directly derived from the nonlinear differential equation. The method was first proposed by Klippel and applied to low frequency[5] and horn-loudspeakers with success [6]. In this paper we present a modified version of the original controller. The original version suppresses the linear part of the selfinductance, an undesirable effect if we want to perform linear equalizing preceding the nonlinear controller. Our version, which follows an alternative derivation, does not has this disadvantage. Starting point is the nonlinear differential equation which is found from substitution of Eq.(2) in Eq.(1)

$$
\begin{aligned}
u_{e}= & \frac{R_{e}}{B l(x)} \mathcal{L}^{-1}\{Z(s)\} * x+\frac{R_{e} k_{t}(x)}{B l(x)} x+ \\
& \frac{d L_{e}(x) i}{d t}+B l(x) \frac{d x}{d t}-\frac{R_{e}}{2 B l(x)} i^{2} \frac{d L_{e}(x)}{d x}
\end{aligned}
$$

We proceed by separation of the linear (desired) part of this differential equation, given by

$$
u=\mathcal{L}^{-1}\left\{\left(Z(s)+k_{t 0}\right)\left(\frac{R_{e}+L_{e 0} s}{B l_{0}}\right)+B l_{0} s\right\} * x
$$

where $B l_{0}, k_{t 0}$ and $L_{e 0}$ are the linear components of the nonlinear elements, i.e. the constant part of their Taylor expansions. Note that in Eq.(9) the linear part of the selfinductance $\left(L_{e 0}\right)$ is included, which is not the case in the
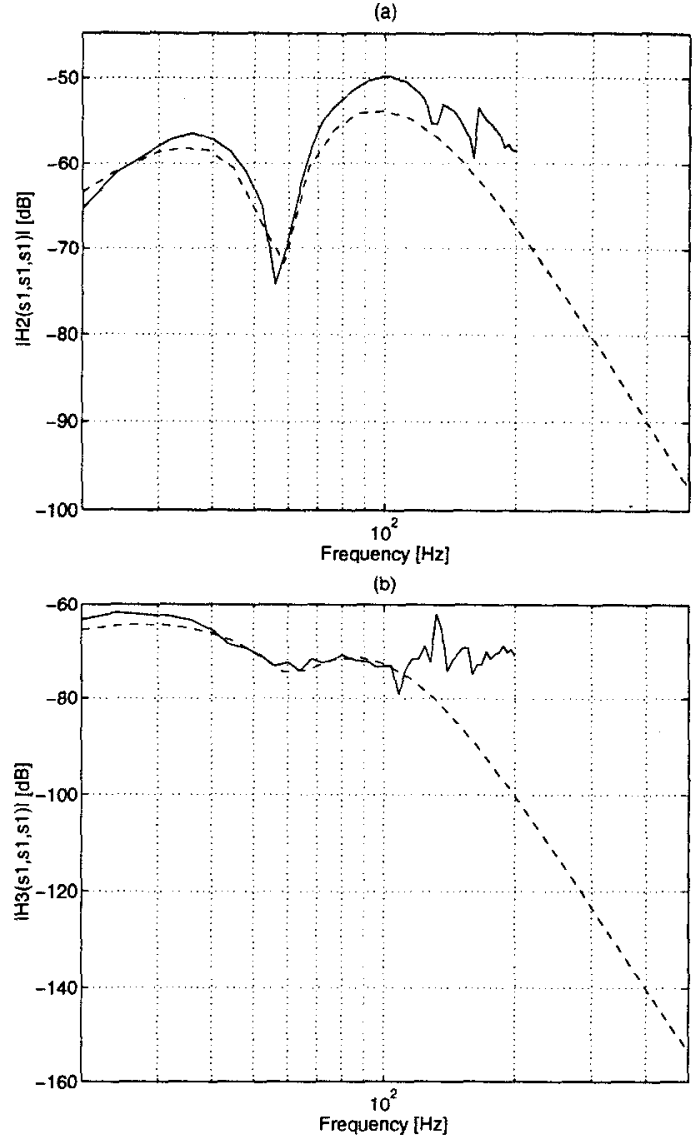

Figure 1. Results of nonlinear parameter optimization of second- (a) and third-order (b) Volterra kernels. Measured microphone voltages (solid lines) are given together with predicted voltages from Volterra model (dashed lines) at a driving level of $7.5 V_{e f f}$.

original derivation [5]. The output of the linearizing controller is now found by defining the voltage $u$ in Eq.(9) as the desired input behavior of the linearized system. The inputoutput description of this controller is then found from substitution of Eq.(9) into Eq.(8), resulting in

$$
\begin{aligned}
u_{o}= & u+N_{B K}(x) x+N_{B 2}(x) \frac{d x}{d t}+N_{B L}(x) i^{2}+ \\
& \frac{d N_{l}(x) i}{d t}+N_{B 1} F_{m}
\end{aligned}
$$

with $u_{o}$ the output of the controller and the static nonlinear operators given by

$$
\begin{aligned}
N_{B K} & =R_{e}\left\{\frac{k_{t}(x)}{B l(x)}-\frac{k_{0}}{B l_{0}}\right\} \\
N_{B 2} & =B l(x)-B l_{0} \\
N_{B L} & =-\frac{R_{e} L_{e x}(x)}{2 B l(x)} \\
N_{l} & =L_{e}(x)-L_{e 0} \\
N_{B 1} & =R_{e}\left\{\frac{1}{B l(x)}-\frac{1}{B l_{0}}\right\}
\end{aligned}
$$




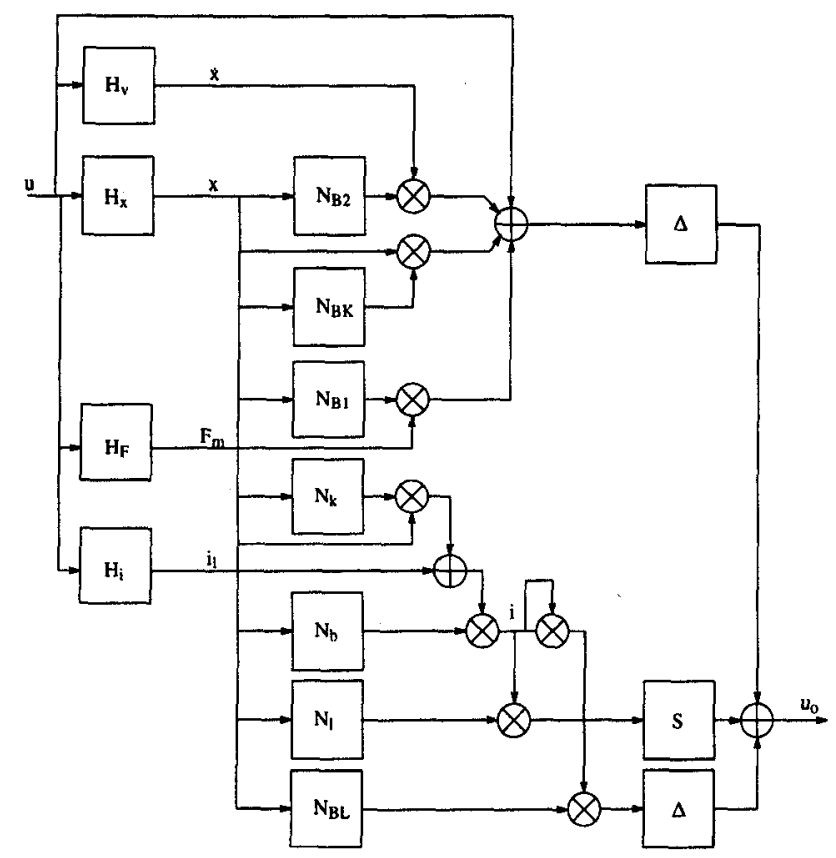

Figure 2. Nonlinear controller according to Eqs.(10)-(13). The $S$-block is the digital differentiator and the $\Delta$-blocks denote constant delay filters to equalize parallel processing paths.

with $L_{e x}(x)$ the first order derivative of $L_{e}(x)$.

Clearly, we need predictions or measurements of the states $x, i, \dot{x}$ and force on the linear mechanical elements $F_{m}$. These are obtained by linear filtering for $x$ and $\dot{x}$ using Eq.(9), resulting in linear filters $H_{x}(s)$ and $H_{v}(s)$ respectively. The current $i$ is determined by nonlinear filtering, using Eq.(2) without the reluctance force, given by

$$
\begin{aligned}
i & =\left\{\mathcal{L}^{-1}\left\{H_{i}(s)\right\} * u+N_{k}(x) x\right\} N_{b}(x) \\
\text { with } \quad N_{k} & =\frac{k_{t}(x)-k_{0}}{R_{e}} \\
N_{b} & =\frac{B l(x)}{B l_{0}}
\end{aligned}
$$

using linear filtering $\left(H_{i}(s)\right)$ to predict the linear current denoted by $i_{l}$. Linear state estimation of the current is not sufficient, as is found from simulations, because of the great sensitivity of the controller towards errors in the current. The force $F_{m}$ is found from linear filtering of the input voltage as well, using the relation

$$
F_{m}=\mathcal{L}^{-1}\left\{Z(s) H_{x}(s)\right\} * u=\mathcal{L}^{-1}\left\{H_{F}(s)\right\} * u .
$$

This results in an algorithm which is digitally implementable using the Bilinear transformation to obtain digital versions of linear filters and using a digital differentiator. The resulting digital controller is schematically depicted in Fig.2. Advantages of the method are the highly transducer related controller structure and its simplicity for higher order nonlinear systems. Disadvantage is the use of the differentiator. At low frequencies $(<500 \mathrm{~Hz})$, however, where distortions are relative large due to the fact that the voice coil excursion is inversely proportional to the squared frequency, these elements can be realized with a sufficient low error.
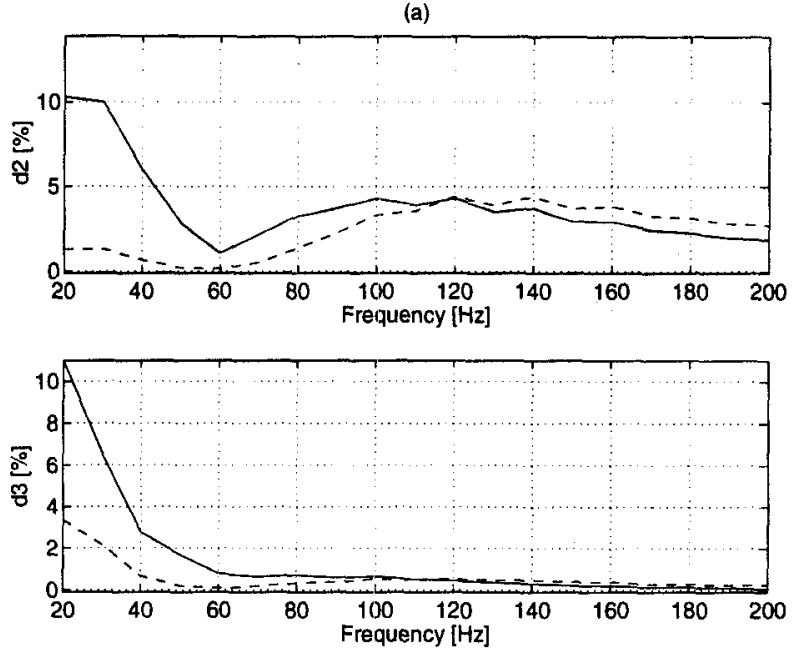

(b)
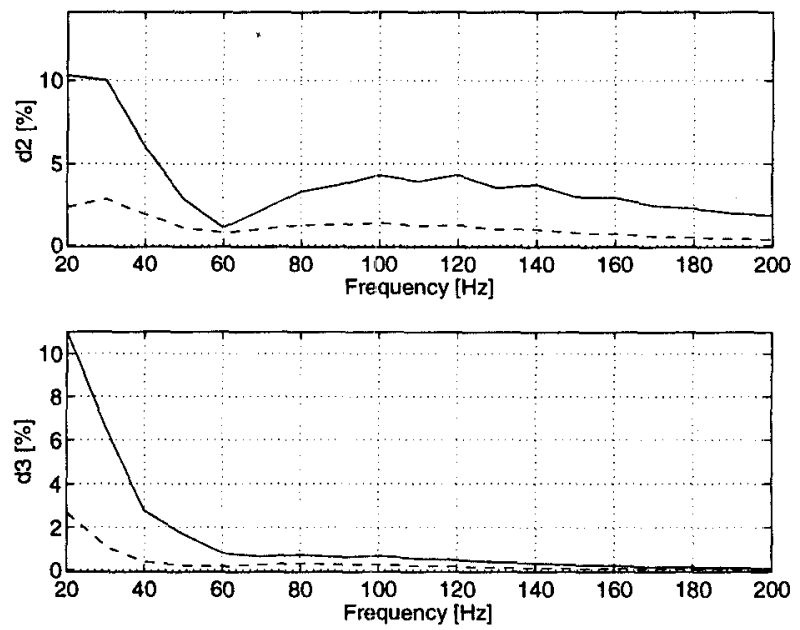

Figure 3. Simulated relative second- $\left(d_{2}\right)$ and third-order $\left(d_{3}\right)$ harmonic distortion at a driving level of $5 V_{e f f}$ without (solid line) and with controller (dashed line). In (a) the original controller [5] is applied and in (b) the new version according to Fig. 2 .

\section{SIMULATION AND EXPERIMENT}

Both controllers, the original version [5] and the new version given by Eqs.(10)- (13), are digitally realized. Digital linear filters in the first two controllers are derived from their continuous frequency domain counterparts and the differentiator is realized using the Simpson integration rule based differentiator [7]. Fractional sample delayers needed in the parallel paths of these controllers are realized by linear interpolators.

\subsection{Simulation}

Using sinusoidal excitations from 20 to $200 \mathrm{~Hz}$ at a driving level of $5 V_{\text {eff }}$ the response of the nonlinear system, with and without controllers applied, is determined. Secondand third-order harmonic distortion are calculated using a 8192 point DFT with an equal length Hanning window. Simulation results with the original controller are given in Fig.3(a) and with the new controller in Fig.3(b). With both controllers distortions are not completely eliminated due to the use of linear state prediction. Clearly seen, however, 
(a)
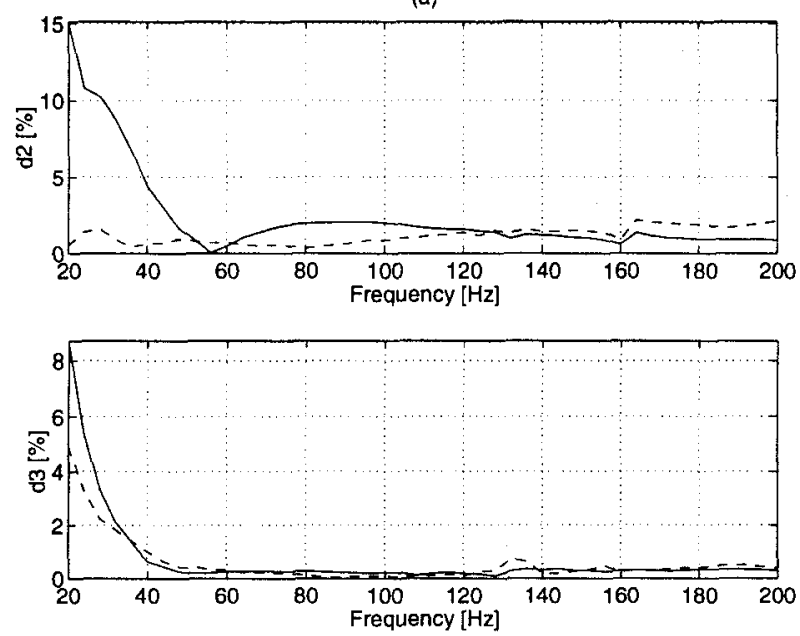

(b)
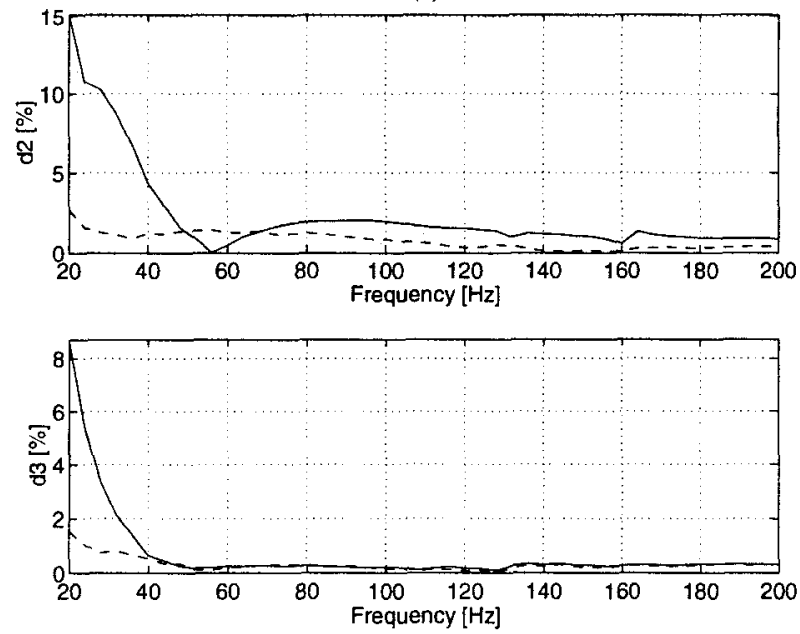

Figure 4. Measured relative second- $\left(d_{2}\right)$ and third-order $\left(d_{3}\right)$ harmonic distortion without (solid line) and with controller (dashed line). In (a) the results with the original controller [5] and in (b) with the new version according to Fig. 2 are given, both at an input voltage of $5 V_{\text {eff }}$.

is the better performance of the new controller compared to the original one which, especially above the $100 \mathrm{~Hz}$, increases second- and third-order distortions. This is due to the equalization of the fundamental response by the original controller at this frequency, which results in an incorrect prediction of the states. From Fig.3(b) we see that distortions are not increased with this controller, although second order distortions at very low frequencies are less reduced. Considering these simulation results we expect a better performance from the new controller.

\subsection{Measurement}

Both controllers are implemented on a general purpose TMS320C30 DSP development board at a sample rate of $15 k H z$. The output of this system drives a highly linear power amplifier which on its turn is connected to a Philips $A D-10202 / W 8$ low frequency loudspeaker in a closed cabinet with a volume of $36 l$, resulting in a resonance frequency of approx. $57 \mathrm{~Hz}$. All distortion measurements are performed in the near-field, avoiding the need for an anechoic chamber, using a microphone and a frequency selective voltage measurement. Results with both controllers are depicted in Fig.4(a)/(b). From Fig.4(a) we observe the resemblance with the results from the simulations with this controller. Especially the second order distortion is increased above $120 \mathrm{~Hz}$. Looking at the results with the new version in Fig.4(b), it is clear that also in practice the performance is indeed better that with the original version. Distortions are even further decreased than predicted by the simulations which is a satisfying result.

\section{CONCLUSIONS}

In this paper we have derived a new linearizing controller to eliminate the electrodynamic transducer nonlinearities. From simulations as well as measurements with a test loudspeaker it is clear that the new version performs better than the original version. Second- and third-order distortions are reduced down to acceptable levels. Main reason of the better performance is the fact that the new controller does not affect the fundamental frequency and therefore makes no error in the state prediction. For loudspeakers with a small self-inductance this is less of a problem and the original controller may suffice. The extra needed computational complexity of the new controller, only two nonlinear static operations and one linear filter, is quite low and therefore not a hindrance for implementation on a general purpose DSP. Although we have derived the nonlinear controller for a loudspeaker in a vented cabinet as well, experimental application of it is left as a topic for future research.

\section{REFERENCES}

[1] M. Schetzen, The Volterra and Wiener theories of nonlinear systems, John Wiley, New York, 1980.

[2] A.J.M. Kaizer, On the design of broadband electrodynamical loudspeakers and multiway loudspeaker systems, Ph.D. thesis Technical University of Eindhoven, 1986.

[3] J.A. Melder and R. Nead, "The convergence of a class of double-rank minimization algorithms," J. Inst. Math. Applic., Vol. 6, pp. 76-90, 1970.

[4] H. Schurer, C.H. Slump and O.E. Herrmann, "Second order Volterra inverses for compensation of loudspeaker nonlinearity," Proceedings of the IEEE ASSP Workshop on applications of signal processing to Audio and Acoustics, New Paltz, pp. 74-78, October 15-18, 1995.

[5] W. Klippel, "The Mirror filter, a new basis for reducing nonlinear distortion and equalizing response in woofer systems," J. Audio Eng. Soc., vol.40 no.9, pp. 675-691, Sept. 1992.

[6] H. Schurer, A.P. Berkhoff, C.H. Slump and O.E. Herrmann, "Modeling and Compensation of Nonlinear Dis. tortion in Horn Loudspeakers," J. Audio Eng. Soc., vol.43 no.7/8, pp. 592-598, Jul./Aug. 1995

[7] M.A. Al-Alaoui, "Novel IIR differentiator from the Simpson integration rule," IEEE transactions on circuits and systems-1: Fundamental theory and applications, vol.41, no.2, pp. 186-187, Feb. 1994. 